\title{
https://doi.org/10.52240/1857-2367.2020.2(21).42 THE ROLE OF AROMATIC PLANTS IN PHYTOCOSMETICS
}

\author{
Maricica COLTUN \\ “Al. Ciubotaru” National Botanical Garden (Institute), \\ Chisinau, Republic of Moldova
}

\begin{abstract}
Along with the industrial production, the popularity of the use of plants in cosmetics has increased in the last decades, because the active compounds extracted from plants and used for the preparation of natural skin care products are not lost or modified during chemical treatments or exposure to high temperatures. The plants used in natural cosmetics are dried naturally and are used as infusion, decoction, tincture, oil or macerated extract, essential oil. In modern cosmetics, natural plant extracts are used a lot and their presence in the ingredient list is a real advantage, since consumers rely on their beneficial effects. In the collection of aromatic plants of the NBGI, several promising species for the cosmetic industry have been introduced and researched, for example: Elsholtzia stauntonii Benth., Rosmarinus officinalis L., Artemisia dracunculus $\mathrm{L}$.
\end{abstract}

Key words: aromatic plant, essential oil, phytocosmetics, active ingredients.

From year to year, there has been an increasing tendency to use products of natural origin, especially plant-derived, in cosmetics, perfumery and aromatherapy. This is due to the fact that such products have virtually no side effects. Hence the need to research plants in all aspects and to obtain convincing evidence of their efficacy in cosmetics. The research conducted at the Botanical Garden also includes the identification of some valuable species of aromatic plants, which can be successfully proposed for implementation in phytocosmetics and aromatherapy.

The amazing health benefits of plants have led to the successful development of phytocosmetics. In order help people maintain the beauty and health of skin, nails or hair, numerous studies have been carried out and it has been found that some plants have astringent and antiseptic effects (chamomile, sage, thyme etc.), others have tonic effect (mint, tarragon etc.) and others have soothing and moisturizing effects on skin (lemon balm, rosemary). To enhance skin regeneration, modern skin care products are made using substances with a chemical structure close to that of the skin, rich in minerals and biostimulators, in order to ensure a biochemical balance.

The species Elsholtzia stauntonii Benth., a species in the Lamiaceae family, introduced in the NBGI and researched as an aromatic and medicinal plant, is of particular interest due to its health benefits. It occurs naturally in China and Pakistan. The plant has a unique decorative aspect at the time of flowering, when it is completely covered with inflorescences of purple flowers, hanging at the ends of the branches. The fragrant leaves, with an extraordinary caraway- and mint-like aroma, can be harvested throughout the summer. E. stauntonii, under the climatic conditions of the Republic of Moldova, accumulates $0.43 \%$ essential oil (1.45 at dry matter), its fragrance note is 4.5 points and it contains high amounts of cinerone (50.8\%) and rosefuran (20.6\%). Other important compounds are: eucalyptol (6.3\%) and $\beta$-caryophyllene $(6.2 \%)$, among the 25 identified ones. The essential oil is fluid, thin and orange and is compatible with all plant fragrances. As for the organoleptic rate, it could refer to fruit and balsamic type, with notes of dried fruits.

Pharmacological studies concerning extracts and pure compounds of E. stauntonii 
have been focused on their antiviral, antibacterial, anti-inflammatory, antioxidant and other activities. Researchers have become increasingly interested in the pharmacological properties of the genus Elsholtzia. A special place is also given to rosemary (Rosmarinus officinalis), an herbaceous plant native to the Mediterranean Basin, with pleasant aroma. In our country, it has been cultivated as a medicinal, spice and ornamental plant. It has antifungal, anti-inflammatory and antioxidant properties. Rosemary oil is a basic ingredient in perfumes and cosmetics. It stimulates hair growth and acts as a conditioner, invigorating the hair, thus restoring its healthy appearance. Rosemary tea slows down the aging process, as it provides a lot of vitamins, minerals and antioxidants, which protect the body from free radicals, moisturize the skin and fight dandruff. Tarragon (Artemisia dracunculus L.) is another aromatic species, it as an herbaceous plant native to Central Asia, known for thousands of years, which is grown in gardens, both for culinary and therapeutic purposes. The main compounds are: estragole, nerol, limonene and various cymenes. It is used in ointments, balms and poultices against pain and muscle cramps. Tarragon essential oil is used to treat eczema, and the extracts of this plant are used as active ingredients in cosmetics. It is considered the ideal weapon to fight digestive disorders, is recommended in respiratory, muscle, gynecological, digestive, hepatobiliary spasms, besides, it is an excellent antiallergenic remedy and antihistamine - a true ally against seasonal allergies caused by pollen, dust, hay etc.

The highlighted species can serve as sources of local raw materials for the production and diversification of the range of natural cosmetics based on plant extracts, for face and body, teeth and mouth, hair, eyes, nails, feet, bath products, soaps, antiperspirant deodorants and personal care products suitable for children.

The research was supported by the NARD through the project "Research and conservation of vascular flora and macromycobiota of the Republic of Moldova", 20.80009.7007.22 (contract Nr. 71/PS/2020).

\section{BIBLIOGRAPHY}

1. Andrea Rasch Brigitte Lotz. Plante aromatice. Bucureşti, 2010, 300 p.

2. Tian,G.H. Chemical constituents in essential oils from Elholtzia and antimicrobial activites. Chinese Herbal Medicines, 5 (2), 2013, 104-108. 